\title{
Waveguide bandgap engineering with an array of superconducting qubits
}

\author{
Jan David Brehm (iD ${ }^{1 凶}$, Alexander N. Poddubny ${ }^{2}$, Alexander Stehli ${ }^{1}$, Tim Wolz ${ }^{1}$, Hannes Rotzinger ${ }^{1,3}$ and Alexey V. Ustinov ${ }^{1,3,4,5}$
}

Waveguide quantum electrodynamics offers a wide range of possibilities to effectively engineer interactions between artificial atoms via a one-dimensional open waveguide. While these interactions have been experimentally studied in the few qubit limit, the collective properties of such systems for larger arrays of qubits in a metamaterial configuration has so far not been addressed. Here, we experimentally study a metamaterial made of eight superconducting transmon qubits with local frequency control coupled to the mode continuum of a waveguide. By consecutively tuning the qubits to a common resonance frequency we observe the formation of super- and subradiant states, as well as the emergence of a polaritonic bandgap. Making use of the qubits quantum nonlinearity, we demonstrate control over the latter by inducing a transparency window in the bandgap region of the ensemble. The circuit of this work extends experiments with one and two qubits toward a full-blown quantum metamaterial, thus paving the way for large-scale applications in superconducting waveguide quantum electrodynamics.

npj Quantum Materials (2021)6:10; https://doi.org/10.1038/s41535-021-00310-z

\section{INTRODUCTION}

The recent advances in the field of quantum information processing has led to a rising demand to explore new systems beyond cavity quantum electrodynamics (QED). One promising candidate is waveguide QED, where quantum systems interact coherently with the mode continuum of a waveguide instead of a cavity. After the pioneering works with single qubits, including the demonstration of resonance flourescence ${ }^{1}$, quantum correlations of light and single photon routers ${ }^{2}$, attention shifted to the realization of multiple qubits coupled to a common waveguide. It was derived ${ }^{3}$ and experimentally verified ${ }^{4}$ that multiple qubits obtain an infinite range photon mediated effective interaction, which can be tuned with the inter-qubit distance $d$. Furthermore, the shared collective excitations are of polaritonic nature with lifetimes ranging from extremely sub- to superradiant relative to the radiative lifetime of the individual qubits ${ }^{5,6}$. The strong intrinsic nonlinearity of the qubits was recently shown to give rise to partially localized polaritons ${ }^{7}$, topological edge states ${ }^{8,9}$, and quantum correlations in the scattered light of the array ${ }^{10}$. The collective quantum properties are exploited in the field of quantum metamaterials ${ }^{11,12}$. Here, the quantum coherence of the constituting qubits is used to engineer a global optical response which depends on their quantum state ${ }^{13-16}$. With respect to quantum information processing, multi-qubit waveguide QED systems could be harnessed in numerous applications such as on demand, highly efficient creation of multi-photon and entangled states ${ }^{17-19}$, storage devices for microwave pulses ${ }^{20}$, atomic mirrors ${ }^{21}$, number-resolved photon detection ${ }^{22}$, slow and even stopped light ${ }^{23}$. Experimentally, waveguide QED systems have been realized on several platforms including atoms ${ }^{24}$, quantum dots coupled to nanophotonic waveguides ${ }^{25}$ and defect centers in diamonds ${ }^{26}$. Even though superconducting qubits feature advantages such as frequency control, high coherence, near perfect extinction and absence of position and number disorder, superconducting multi-qubit waveguide QED systems have been studied only recently to some extend ${ }^{27,28}$.

Here, we investigate the mode spectrum of a metamaterial formed by a densely spaced array of eight superconducting transmon qubits coupled to a coplanar waveguide. By employing dedicated flux-bias lines for each qubit, we establish control over their transition frequencies. Thus we are able to alter the number of resonant qubits $N$ at will, allowing us to observe super- and subradiant modes as well as the gradual formation of a bandgap. Our spectroscopic measurements show, that through this control the global optical susceptibility of the metamaterial can be tuned. A demonstration for the collective Autler-Townes splitting (ATS) of eight qubits is presented, which marks an important step toward the implementation of quantum memories.

\section{RESULTS AND DISCUSSION \\ Circuit design and properties}

The sample under investigation is depicted in Fig. 1(a). The spacing $d$ between adjacent qubits is $400 \mu \mathrm{m}$, which is smaller than the corresponding wavelength $\lambda$ at all accessible frequencies $\left(\varphi=\frac{2 \pi}{\lambda} d=0.05-0.16\right)$. The dense spacing is chosen in order to increase the width of the expected bandgap of $\Delta \omega=\Gamma_{10} / \varphi \gg \Gamma_{10}$ and to fulfil the metamaterial limit of subwavelength dimensions ${ }^{29}$. Here $\Gamma_{10}$ is the radiative decay rate of the individual qubits into the waveguide. The qubits are overcoupled, ensuring a multimode Purcell-limited rate $\Gamma_{10} / 2 \pi \approx 6.4 \mathrm{MHz}$ for high extinction and, simultaneously, better subradiant state visibility. All qubits are individually frequency controllable between 3 and $8 \mathrm{GHz}$ by changing the critical currents of the qubit SQUIDs with local fluxbias lines. We compensate unwanted magnetic crosstalk between the flux-bias lines and neighbouring qubits by extracting and diagonalising the full mutual inductance matrix $M$ (see Methods). This allows us to counteract the parasitic crosstalk flux by sending appropriate currents to all qubits, which are not actively tuned.

\footnotetext{
${ }^{1}$ Physikalisches Institut, Karlsruhe Institute of Technology, 76131 Karlsruhe, Germany. ${ }^{2}$ loffe Institute, St. Petersburg, Russia194021. ${ }^{3}$ Institute for Quantum Materials and Technologies, Karlsruhe Institute of Technology, 76021 Karlsruhe, Germany. ${ }^{4}$ National University of Science and Technology MISIS, Moscow, Russia119049. ${ }^{5}$ Russian Quantum Center, Skolkovo, Moscow, Russia143025. ${ }^{\circledR}$ email: jan.brehm@kit.edu
} 


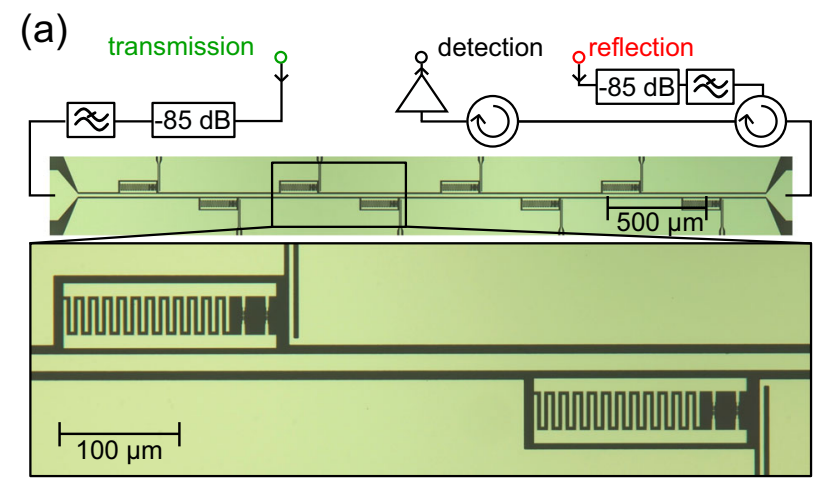

(b)

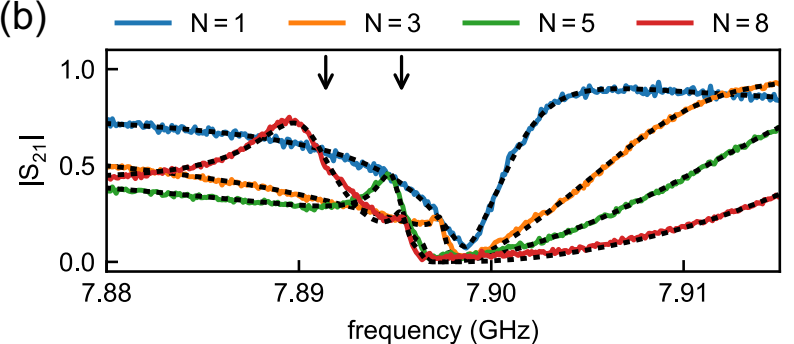

Fig. 1 Sample and transmission. a Optical micrograph of the metamaterial. It is composed of eight superconducting transmon qubits capacitively coupled to a coplanar waveguide. Local flux-bias lines provide individual qubit frequency control in the range 3-8 GHz. b Transmission $\left|S_{21}\right|$ for different numbers $N$ of resonant qubits at $\omega_{\mathrm{r}} / 2 \pi=7.898 \mathrm{GHz}$ and low drive powers. With increasing $N$, the emergence of subradiant states (visible as peaks in transmission) can be observed. Black dotted lines are fits to the expected transmission using a transfer matrix calculation. Black arrows mark calculated frequencies of the two brightest subradiant states for $N=8$.

With this calibration scheme we achieve precise control over the individual qubit frequencies. We estimate the residual crosstalk to be smaller than $10^{-3}$. The effective Hamiltonian of this system, after formally tracing out photonic degrees of freedom and applying the Markov approximation, is described by ${ }^{6}$,

$$
\frac{H_{\text {eff }}}{\hbar}=\sum_{j}^{8}\left(\omega_{j} b_{j}^{\dagger} b_{j}+\frac{X_{j}}{2} b_{j}^{\dagger} b_{j}^{\dagger} b_{j} b_{j}\right)+\mathrm{i} \frac{\Gamma_{10}}{2} \sum_{k \neq j}^{8} b_{k}^{\dagger} b_{j} \mathrm{e}^{-\mathrm{i} \frac{\omega d}{c}|k-j|},
$$

with the bosonic creation operator $b_{j}^{\dagger}|0\rangle=\left|\mathrm{e}_{j}\right\rangle$, exciting the $j$-th qubit at frequency $\omega_{j}$; where we assume a $\propto e^{+i \omega t}$ time dependence of the excitations. Here, $X_{j} / 2 \pi$ is the qubit anharmonicity for which we find spectroscopically weakly varying values around $-275 \mathrm{MHz}$. The last term of $H_{\text {eff }}$ describes the effective qubit-qubit coupling. Due to the specific choice of small $d$ its imaginary part dominates over the real part, leading to a suppressed exchange-type interaction between the qubits. The expected eigenfrequencies $\omega_{\xi}$ of $H_{\text {eff }}$ in the single excitation limit and $X \rightarrow 0$ are shown in Fig. 2(a).

\section{Bandstructure and collective metamaterial excitations}

First, we characterize the mode spectrum of the metamaterial in dependence on the number of resonant qubits $N$ by measuring the transmission coefficient $S_{21}(\omega)$ while the qubits are consecutively tuned to a common resonance frequency at $\omega_{\mathrm{r}} / 2 \pi=$ $7.898 \mathrm{GHz}$, compare Fig. 1 (b) and Fig. 2 (a). The incident photon power $P_{\text {inc }}$ is kept below the single photon level $\left(P_{\text {inc }} \ll \hbar \omega \Gamma_{10}\right)$ to avoid saturation of the qubits. For a single qubit, the well known resonance-fluorescence was observed as a single dip in transmis$\operatorname{sion}^{1}$. By fitting the complex transmission data to the expected transmission function $S_{21}(\omega)$ (see Methods) the individual
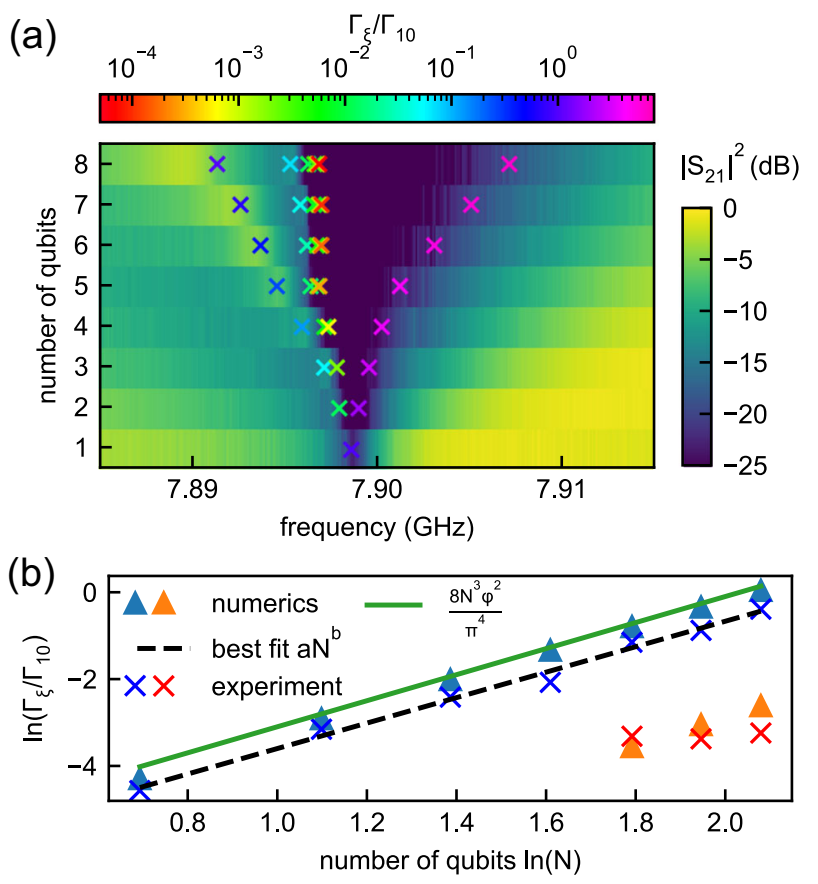

Fig. 2 Bandgap and polariton relaxation rates. a Dependence of absolute transmission $\left|S_{21}\right|^{2}$ on the number of resonant qubits $N$. Crosses mark calculated eigenfrequencies $\omega_{\xi}$ of $H_{\text {eff }}$ and their corresponding radiative decay rates $\Gamma_{\xi} / \Gamma_{10}$ (color coded). With increasing $N$ a bandgap of strongly supressed transmission opens up. b Measured radiative decay rates $\Gamma_{\xi}$ of the brightest (blue crosses) and second brightest (red crosses) subradiant states. Orange and blue solid triangles are the corresponding calculated rates, the green line is the analytical result $\Gamma_{\xi}=8 N^{3} \varphi^{2} / \pi^{4}$. For the brightest subradiant state, a fit to a power-law with exponent $b=$ $2.93 \pm 0.13$ (black dashed line) confirms the scaling of $\Gamma_{\xi} \propto N^{3}$.

coherence properties of all eight qubits at $\omega_{\mathrm{r}}$ can be extracted (see Supplementary Note 1). We find the average radiative rates $\Gamma_{10} / 2 \pi=6.4 \mathrm{MHz}$ and the intrinsic non-radiative rates $\Gamma_{\mathrm{nr}} / 2 \pi=$ $240-560 \mathrm{kHz}$. For $N \geq 2$ resonant qubits the system obtains multiple eigenmodes and the super- and subradiant polariton modes start to emerge. The superradiant mode is manifested as a wide transmission dip above $\omega_{r}$, the subradiant modes can be identified as transmission peaks below $\omega_{\mathrm{r}}$. We note that the peak shape is created by Fano-interference of the sub- and the superradiant modes (see Supplementary Note 5), which also causes the calculated eigenfrequencies of $H_{\text {eff }}$ to not exactly coincide with the maximum of the peaks in Fig. 1 (b) and Fig. 2(a). For $N \geq 6$ qubits a second darker subradiant mode is visible between $\omega_{r}$ and the brightest subradiant mode. The limiting factor for the observation of the subradiant states is the intrinsic qubit coherence as given by $\Gamma_{n r}$, setting an upper threshold for the maximum observable lifetime. Darker subradiant states with $\Gamma_{\xi}<$ $\Gamma_{\text {nr }}$ decay in the qubits into dielectric channels or dephase due to flux noise in the SQUIDs before they are remitted into the waveguide. The observed transmission coefficient $\left|S_{21}\right|$ is in good agreement with the calculated transmission based on a transfer matrix approach ${ }^{30}$. The asymmetric lineshape of the resonances is a parasitic effect caused by interference of the signal with low- $Q$ standing waves in the cryostat ${ }^{31}$. We account for this effect in the transfer matrix calculation by adding semi-reflective inductances in front and after the qubit array. As shown in Fig. 2(a), a frequency region of strongly suppressed transmission with $\left|S_{21}\right|^{2}<-25 \mathrm{~dB}$ is opening up above $\omega_{r}$ with increasing $N$. This effect is associated with the emergence of a polaritonic bandgap, where the effective refractive index becomes purely imaginary, as expected for any kind of resonant periodic structures ${ }^{29,32}$. For $N=8$ qubits we 
extract a bandgap bandwidth of $\Delta \omega \approx 1.9 \Gamma_{10}$. Compared to the expected bandgap width of $\Delta \omega=\Gamma_{10} / \varphi \approx 6.3 \Gamma_{10}$ of the structure for $N \rightarrow \infty$ this places our system size in the transitioning regime between a single atom and a fully extended metamaterial with a continuous mode spectrum. The radiative decay rates $\Gamma_{\xi}$ of the observed subradiant states shown in Fig. 2(b) are extracted by fitting Lorentzians to the corresponding modes in the reflection data (not shown). It can be generally shown, that $\left|S_{21}\right|^{2}$ indeed obtains a Lorentzian shape in the vicinity of each $\omega_{\xi}^{30,33,34}$. From a fit to a power law $\propto N^{b}$ with exponent $b=2.93 \pm 0.13$ we find that the rate of the brightest subradiant states scales with $\Gamma_{\xi} \propto N^{3}$, which we also find analytically for densely packed qubit structures with $\varphi \ll 1$ from $H_{\text {eff }}$ (see Supplementary Note 4). The found scaling is the complementary asymptotic of the theoretically predicted $\Gamma_{\varepsilon} \propto N^{-3}$ law for the darkest subradiant modes in references ${ }^{5,6,32}$. Small deviations between calculated and measured values of $\Gamma_{\xi}$ are caused by imperfect qubit tuning and distortions of the observed reflection coefficient due to the microwave background.

The control over the mode spectrum can be further elaborated with the off-resonant situation, where one qubit has a finite detuning $\Delta$ from the common resonance frequency $\omega_{r}$ of the residual qubits by sweeping it through the common resonance as shown in Fig. 3(a). Here, qubit 8 is tuned through the collective resonance of qubits $1-7$. For large detunings $|\Delta| \gg \Gamma_{10}$ the modes of the ensemble and qubit 8 are not hybridized (not shown). For smaller detunings an additional partially hybridized subradiant mode appears, which becomes for $\Delta \approx 0$ the brightest subradiant state of the fully hybridized 8-qubit system. The result is in good agreement with the transfer matrix calculation and direct diagonalization of $H_{\text {eff }}$ as shown in Fig. 3(b). The level repulsion between the eigenstates is caused by the residual exchange-type interaction between the qubits due to the finite inter-qubit distance $d$. In Fig. 3 there are several blind spots where the subradiant states turn completely dark, occurring when the
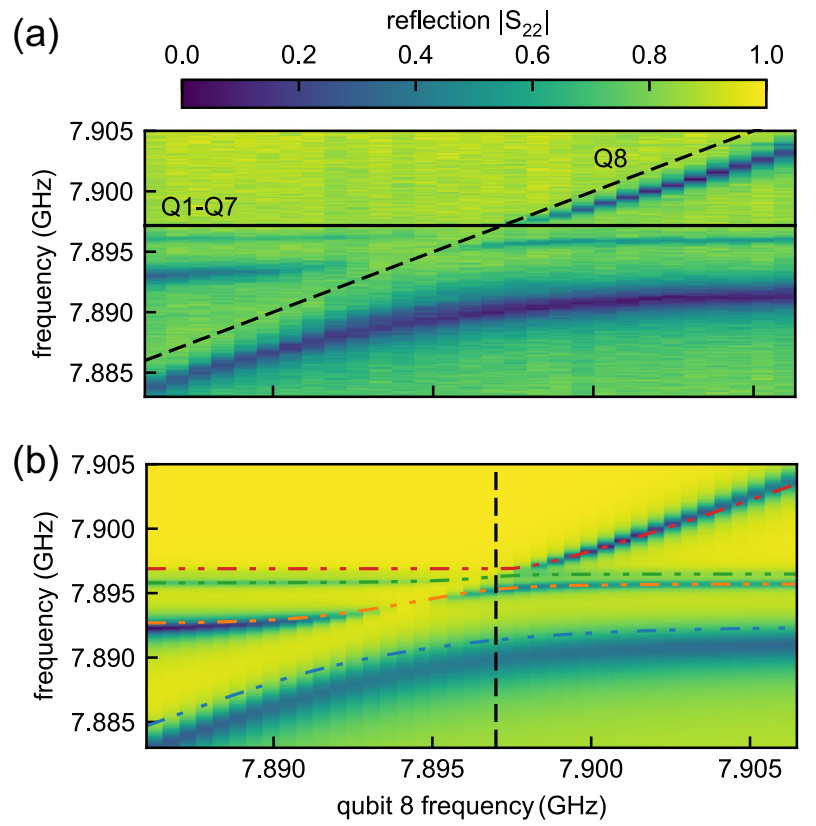

Fig. 3 Off-resonant qubits. a Measured absolute reflection $\left|S_{22}\right|$ for $N=7$ resonant qubits at $\omega_{\mathrm{r}} / 2 \pi=7.897 \mathrm{GHz}$ (solid black line) and qubit 8 being tuned through the collective resonance (black dashed line). b Calculated reflection with transfer matrix method and relevant eigenfrequencies of $H_{\text {eff }}$ (dash dotted lines) show good agreement with experimental result in (a). The vertical line indicates the resonance with $\omega_{8}=\omega_{r}$, corresponding to the situation observed in Fig. 1. frequency of the detuned qubit matches the frequency of a dark mode. This is explained by the Fano-like interferences ${ }^{35}$ between the detuned qubit resonance and the modes of the resonant qubits, which are analyzed in more detail in the Supplementary Note 5 .

\section{Collective Autler-Townes splitting}

The intrinsic quantum nonlinearity of the system due to the anharmonic nature of the qubits can be probed by increasing the microwave power beyond the single photon regime $\left(P_{\text {inc }}>\hbar \omega \Gamma_{10}\right)$. At higher power the $0 \rightarrow 1$ transition of a single qubit will start to saturate and transmission at resonance will increase back to unity ${ }^{1}$, see Fig. $4(\mathrm{~b})$. We could experimentally verify the prediction of the saturation of an ensemble of resonant qubits at higher drive rates of refs. ${ }^{1,3}$, which can be observed in Fig. 4(a). We point out that all spectroscopic features of the metamaterial, such as superand subradiant modes as well as the bandgap, are saturable. The power $P_{50 \%}$ needed to saturate the transmission at $\omega_{r}$ to $50 \%$ $\left(\left|S_{21}\right|^{2}=0.5\right)$ grows with an approximate scaling $\propto \ln (\mathrm{N})$. The observed quantum nonlinear behavior establishes a border to previously studied metamaterials consisting of harmonic resonators rather than qubits ${ }^{36}$. Furthermore the anharmonic level structure of the transmon can be used to electromagnetically open a transparency window around the frequency of the $0 \rightarrow 1$ transition, by employing the ATS ${ }^{37}$. As indicated in Fig. 4(c), a coherent control tone with Rabi strength $\Omega_{c}$ and frequency $\omega_{c}$ drives the $1 \rightarrow 2$ transition, while a weak microwave tone with $\Omega_{\mathrm{p}} \ll \Omega_{\mathrm{c}}$ is probing the transmission at frequencies $\omega_{\mathrm{p}}$ around the $0 \rightarrow 1$ transition. The control tone is dressing the first qubit level, creating two hybridized levels, which are separated proportionally to its amplitude, and is therefore creating a transparency window with respect to the probe. To the best of our knowledge this effect was so far only demonstrated for a single qubit in superconducting waveguide QED, leading to applications like single photon routers ${ }^{2}$. Here, we observe the collective ATS for up to $N=$ 8 resonant qubits (Fig. 4(d)). Analogously to the single qubit case, the observed level splitting is proportional to $\Omega_{\mathrm{c}}$. At control tone powers $>-110 \mathrm{dBm}$ the bandgap is rendered fully transparent and transmission close to unity is restored. In the experiment we find slightly smaller than unity values of $\left|S_{21}\right| \approx 0.75$ due to the interference of the signal with the microwave background. In the bandstructure picture the collective ATS can be understood as the dressed states of the individual qubits giving rise to two independent Bloch bands ${ }^{38}$. Therefore, the two branches of the collective ATS with supressed transmission are collectively broadened bandgaps and have a larger linewidth than the dressed states of the single qubit ATS. As pointed out in reference $^{38}$ the collective ATS demonstrates active control over the bandstructure of the metamaterial via the parameters $\omega_{\mathrm{c}}$ and $\Omega_{c}$. The observed splittings are in agreement with a transfer matrix calculation and a full master equation simulation (see Supplementary Note 6). Minor deviations are caused by imperfect qubit tuning and differing qubit anharmonicities of about $\sigma_{X} \approx 10 \mathrm{MHz}$. We argue here that the single photon router concept with a single qubit can significantly be improved with multiple qubits, which form a much wider stop-band with higher saturation power, thus permitting to route also multiple photons.

In conclusion, we demonstrated a fully controllable quantum metamaterial consisting of eight densely packed transmon qubits coupled to a waveguide. Such intermediate system size combined with individual qubit control allowed us to explore the transition from a single mode regime to a continuous band spectrum. By tuning the qubits consecutively to resonance, we observed the emergence of a polaritonic bandgap, and confirmed the scaling of the brightest dark mode decay rate with the qubit number. Active control over the band structure of the ensemble was demonstraded by inducing a transparency window in the bandgap 

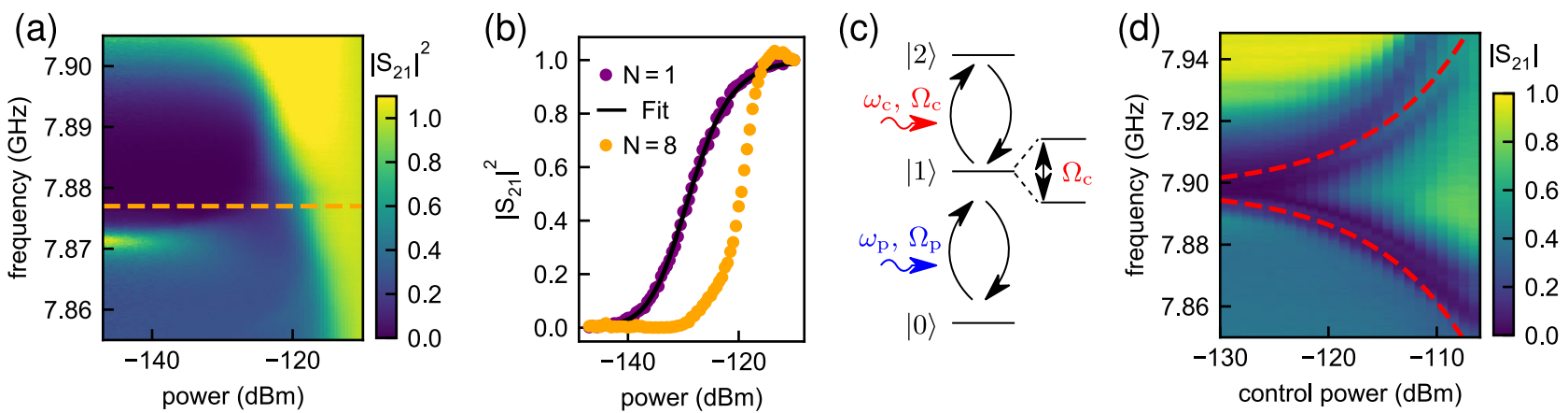

Fig. 4 Power saturation and Autler-Townes splitting. a Absolute power transmission $\left|S_{21}\right|^{2}$ for $N=8$ resonant qubits shows saturation with increasing power due to the anharmonic level structure of the transmons. $\mathbf{b}$ Comparison between the saturation of $\left|S_{21}\right|^{2}$ at $\omega_{\mathrm{r}}$ for a single qubit and $N=8$ qubits. c Schematic illustration of the Autler-Townes effect for a ladder-type three-level system. A control tone is driving the $1 \rightarrow 2$ transition with Rabi strength $\Omega_{c}$ and frequency $\omega_{c}$, while a weak tone is probing the transmission of the $0 \rightarrow 1$ transition. $\mathbf{d}$ Experimental demonstration of the collective Autler-Townes splitting for $N=8$. The red dashed line marks the fitted level separation to $\Omega_{\text {c. }}$.

region, using the Autler-Townes effect. Our work promotes further research with higher qubit numbers to realize a long-living quantum memory.

\section{METHODS}

\section{Fabrication}

The sample is fabricated with two consecutive lithography steps from thermally evaporated aluminum in a Plassys MEB550s shadow evaporator on a $500 \mu \mathrm{m}$ sapphire substrate. In a first step solely the qubits are patterned with $50 \mathrm{keV}$ electron beam lithography. The Josephson junctions are patterned with a bridge-free fabrication technique ${ }^{39}$ using a PMMA/ PMMA-MAA double resist stack. Before double-angle evaporation, the developed resist stack is cleaned for $6 \mathrm{~min}$ with an oxygen-plasma to remove resist residuals in the junction area to reduce the impact of junction aging ${ }^{39}$. In a second optical lithography step we pattern the CPWwaveguide and the ground-plane in a liftoff-process on $\mathrm{S} 1805$ photo resist. The SQUIDs are formed by two Josephson junctions, enclosing an area of $560 \mu \mathrm{m}^{2}$. By design, the junction areas are 0.12 and $0.17 \mu \mathrm{m}^{2}$ with a designed asymmetry of $17 \%$.

\section{Calibration of magnetic crosstalk}

We extract the full $8 \times 8$ mutual inductance matrix $M$ between the qubits and the bias coils in 28 consecutive measurements. For that, the transmission through the chip is observed at a fixed frequency while tuning the currents of two qubits $I_{x}$ and $I_{y}$ such that they get tuned through the observation frequency. Fitting the slope of the observed qubit traces gives access to $M_{x y} / M_{x x}$ and $M_{y x} / M_{y y}$. When $M$ is known, compensation currents which are send to all qubits which are not actively tuned, can be calculated to compensate unwanted crosstalk. We estimate the residual crosstalk to be smaller than $0.1 \%$. Further information is provided in the Supplementary Note 2 .

\section{Normalization of spectroscopic data}

We normalize the transmission data by dividing the raw data $S_{21}^{\text {raw }}$ by the transmission data at high powers $S_{21}^{\text {sat }}: S_{21}^{\text {calib }}(\omega)=S_{21}^{\text {raw }}(\omega) /\left(a S_{21}^{\text {sat }}(\omega)\right)$, where $a \approx 1$ is a constant factor accounting for weak fluctuations of the amplifier gain. Reflection data is normalized by dividing the raw data $S_{22}^{\text {raw }}$ with its maximum value at the qubit resonance frequency $f_{r}$ : $S_{22}^{\text {calib }}=S_{22}^{\text {raw }} / S_{22}^{\text {raw }}\left(f_{\mathrm{r}}\right)$. This approximation is justified for the sample under investigation since the extinction of single and multiple qubits is very close to 1. A more rigorous normalization of the data based on energy conservation $\left|S_{21}\right|^{2}+\left|S_{11}\right|^{2} \approx 1$ is not applicable here, due to differing signal paths for reflection and transmission measurements, as pointed out in the main text. In addition, in any experimental system the insertion losses due to impedance mismatches of the feedline can not be avoided. Since they are in general not symmetric on both sides of the sample, $\left|S_{11}\right| \neq\left|S_{22}\right|$ and therefore $\left|S_{21}\right|^{2}+\left|S_{22}\right|^{2} \neq 1$.

\section{Characterization of individual qubits}

The amplitude transmission coefficient of a driven two-level system sidecoupled to a waveguide $S_{21}$ is given by ${ }^{2}$ :

$$
S_{21}=1-\frac{\Gamma_{10}}{2 \gamma_{10}} \frac{1-\mathrm{i} \frac{\omega-\omega_{\mathrm{r}}}{\gamma_{10}}}{1+\left(\frac{\omega-\omega_{\mathrm{r}}}{\gamma_{10}}\right)^{2}+\frac{\Omega_{\mathrm{p}}^{2}}{\left(\Gamma_{10}+\Gamma_{1}\right) \gamma_{10}}}
$$

The decoherence rate $\gamma_{10}=\Gamma_{10} / 2+\Gamma_{n r}$ is the sum of radiative decay $\Gamma_{10}$ and non-radiative decay rates $\Gamma_{n r}=\Gamma_{\Phi}+\Gamma_{/} / 2$. Here $\Gamma_{\Phi}$ accounts for pure

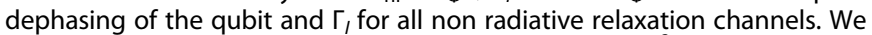
define the extinction coefficient as $1-\left(1-\Gamma_{10} / 2 \gamma_{10}\right)^{2}$, measuring the suppression of power-transmission at very low drive powers. A circle fitting procedure $^{40}$ is used to fit equation (2) to the measured complex transmitted signal $S_{21}$ in the limit of weak driving $\Omega_{\mathrm{p}}^{2} \ll\left(\Gamma_{10}+\Gamma_{1}\right) \gamma_{10}$. The decoherence rates of the individual qubits and further details on the fitting procedure are provided in the Supplementary Note 1.

\section{DATA AVAILABILITY}

The data that support the findings of this study are available from the corresponding author upon reasonable request.

Received: 14 September 2020; Accepted: 28 December 2020; Published online: 04 February 2021

\section{REFERENCES}

1. Astafiev, O. et al. Resonance fluorescence of a single artificial atom. Science 327, 840-843 (2010)

2. Hoi, I.-C. et al. Microwave quantum optics with an artificial atom in onedimensional open space. N. J. Phys. 15, 025011 (2013).

3. Lalumière, K. et al. Input-output theory for waveguide QED with an ensemble of inhomogeneous atoms. Phys. Rev. A. 88, 043806 (2013).

4. Loo, A. F. V. et al. Photon-mediated interactions between distant artificial atoms. Science 342, 1494-1496 (2013).

5. Zhang, Y.-X. \& Mølmer, K. Theory of subradiant states of a one-dimensional twolevel atom chain. Phys. Rev. Lett. 122, 203605 (2019).

6. Albrecht, A. et al. Subradiant states of quantum bits coupled to a onedimensional waveguide. N. J. Phys. 21, 025003 (2019).

7. Zhong, J. et al. Photon-mediated localization in two-level qubit arrays. Phys. Rev. Lett. 124, 093604 (2020)

8. Ke, Y. et al. Radiative topological biphoton states in modulated qubit arrays. Phys. Rev. Res. 2, 033190 (2020).

9. Poshakinskiy, A. V. et al. Quantum Hall phase emerging in an array of atoms interacting with photons. Preprint at https://arxiv.org/abs/2003.08257 (2020).

10. Fang, Y.-L. L., Zheng, H. \& Baranger, H. U. One-dimensional waveguide coupled to multiple qubits: photon-photon correlations. EPJ Quantum Technol. 1, 3 (2014).

11. Rakhmanov, A. L., Zagoskin, A. M., Savel'ev, S. \& Nori, F. Quantum metamaterials: electromagnetic waves in a Josephson qubit line. Phys. Rev. B 77, 144507 (2008).

12. Macha, $P$. et al. Implementation of a quantum metamaterial using superconducting qubits. Nat. Commun. 5, 5146 (2014). 
13. Asai, H., Savel'ev, S., Kawabata, S. \& Zagoskin, A. M. Effects of lasing in a onedimensional quantum metamaterial. Phys. Rev. B. 91, 134513 (2015).

14. Ivić, Z., Lazarides, N. \& Tsironis, G. P. Qubit lattice coherence induced by electromagnetic pulses in superconducting metamaterials. Sci. Rep. 6, 29374 (2016).

15. Bamba, M., Inomata, K. \& Nakamura, Y. Superradiant phase transition in a superconducting circuit in thermal equilibrium. Phys. Rev. Lett. 117, 173601 (2016).

16. Asai, H., Kawabata, S., Savel'ev, S. E. \& Zagoskin, A. M. Quasi-superradiant soliton state of matter in quantum metamaterials. Eur. Phys. J. B. 91, 30 (2018).

17. Paulisch, V., Kimble, H. J., Cirac, J. I. \& González-Tudela, A. Generation of singleand two-mode multiphoton states in waveguide QED. Phys. Rev. A. 97, 053831 (2018).

18. González-Tudela, A., Paulisch, V., Kimble, H. \& Cirac, J. Efficient multiphoton generation in waveguide quantum electrodynamics. Phys. Rev. Lett. 118, 213601 (2017).

19. Zhang, X. H. \& Baranger, H. U. Heralded Bell state of dissipative qubits using classical light in a waveguide. Phys. Rev. Lett. 122, 140502 (2019).

20. Leung, P. M. \& Sanders, B. C. Coherent control of microwave pulse storage in superconducting circuits. Phys. Rev. Lett. 109, 253603 (2012).

21. Chang, D. E., Jiang, L., Gorshkov, A. V. \& Kimble, H. J. Cavity qed with atomic mirrors. N. J. Phys. 14, 063003 (2012).

22. Malz, D. \& Cirac, J. I. Nondestructive photon counting in waveguide qed. Phys. Rev. Res. 2, 033091 (2020).

23. Everett, J. L., Higginbottom, D. B., Campbell, G. T., Lam, P. K. \& Buchler, B. C. Stationary light in atomic media. Adv. Quantum Technol. 2, 1800100 (2019).

24. Solano, P., Barberis-Blostein, P., Fatemi, F. K., Orozco, L. A. \& Rolston, S. L. Superradiance reveals infinite-range dipole interactions through a nanofiber. Nat. Commun. 8, 1857 (2017).

25. Javadi, A. et al. Single-photon non-linear optics with a quantum dot in a waveguide. Nat. Commun. 6, 8655 (2015).

26. Sipahigil, A. et al. An integrated diamond nanophotonics platform for quantumoptical networks. Science 354, 847-850 (2016).

27. Mirhosseini, M. et al. Cavity quantum electrodynamics with atom-like mirrors. Nature 569, 692-697 (2019).

28. Kim, E. et al. Quantum electrodynamics in a topological waveguide. Preprint at http://arxiv.org/abs/2005.03802 (2020).

29. Ivchenko, E. L. Excitonic polaritons in periodic quantum-well structures. Sov. Phys. Sol. State 33, 1344-1346 (1991)

30. Asenjo-Garcia, A., Moreno-Cardoner, M., Albrecht, A., Kimble, H. \& Chang, D. Exponential improvement in photon storage fidelities using subradiance and "selective radiance" in atomic arrays. Phys. Rev. X 7, 031024 (2017).

31. Khalil, M. S., Stoutimore, M. J. A., Wellstood, F. C. \& Osborn, K. D. An analysis method for asymmetric resonator transmission applied to superconducting devices. J. Appl. Phys. 111, 054510 (2012).

32. Tsoi, T. S. \& Law, C. K. Quantum interference effects of a single photon interacting with an atomic chain inside a one-dimensional waveguide. Phys. Rev. A. 78 063832 (2008).

33. Ivchenko, E. L., Nesvizhskii, A. I. \& Jorda, S. Bragg reflection of light from quantumwell structures. Phys. Solid State 36, 1156-1161 (1994).

34. Kosobukin, V. A. \& Poddubny, A. N. Exciton-polariton absorption in periodic and disordered quantum-well chains. Phys. Solid State 49, 1977-1987 (2007).

35. Limonov, M. F., Rybin, M. V., Poddubny, A. N. \& Kivshar, Y. S. Fano resonances in photonics. Nat. Photonics 11, 543-554 (2017).

36. Mirhosseini, M. et al. Superconducting metamaterials for waveguide quantum electrodynamics. Nat. Commun. 9, 3706 (2018).

37. Abdumalikov, A. A. et al. Electromagnetically induced transparency on a single artificial atom. Phys. Rev. Lett. 104, 193601 (2010).

38. Witthaut, D. \& Sørensen, A. S. Photon scattering by a three-level emitter in a onedimensional waveguide. N. J. Phys. 12, 043052 (2010).

39. Lecocq, F. et al. Junction fabrication by shadow evaporation without a suspended bridge. Nanotechnology 22, 315302 (2011).

40. Probst, S., Song, F. B., Bushev, P. A., Ustinov, A. V. \& Weides, M. Efficient and robust analysis of complex scattering data under noise in microwave resonators. Rev. Sci. Instrum. 86, 024706 (2015).

\section{ACKNOWLEDGEMENTS}

This work has received funding from the Deutsche Forschungsgemeinschaft (DFG) by the Grant No. US 18/15-1, the European Union's Horizon 2020 Research and Innovation Programme under Grant Agreement No. 863313 (SUPERGALAX), and by the Initiative and Networking Fund of the Helmholtz Association, within the Helmholtz Future Project 'Scalable solid state quantum computing'. We acknowledge financial support from Studienstiftung des Deutschen Volkes (J.D.B.), Landesgraduiertenförderung-Karlsruhe (A.S.) and Helmholtz International Research School for Teratronics (T.W.). Analysis of subradiant mode lifetimes, performed by A.N.P., has been supported by the Russian Science Foundation Grant 20-12-00194. Basic concepts for this work were developed with the financial support from the Russian Science Foundation (contract No. 16-12-00095). A.V.U. acknowledges partial support from the Ministry of Education and Science of the Russian Federation in the framework of the Increase Competitiveness Program of the National University of Science and Technology MISIS (contract No. K2-2020-022).

\section{AUTHOR CONTRIBUTIONS}

J.D.B. fabricated the devices supported by H.R. J.D.B. performed the measurements with support of A.S. and T.W. A.S. developed concepts for the crosstalk calibration. A V.U. and H.R. setup the measurement facility. J.D.B. analyzed the data. A.N.P. performed the calculations on Fano-interference and linewidth-scaling. J.D.B. and A N.P. wrote the paper. All authors contributed to the discussion. The project was supervised by H.R. and A.V.U.

\section{FUNDING}

Open Access funding enabled and organized by Projekt DEAL.

\section{COMPETING INTERESTS}

The authors declare no competing interests.

\section{ADDITIONAL INFORMATION}

Supplementary information The online version contains supplementary material available at https://doi.org/10.1038/s41535-021-00310-z.

Correspondence and requests for materials should be addressed to J.D.B.

Reprints and permission information is available at http://www.nature.com/ reprints

Publisher's note Springer Nature remains neutral with regard to jurisdictional claims in published maps and institutional affiliations.

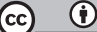

Open Access This article is licensed under a Creative Commons Attribution 4.0 International License, which permits use, sharing, adaptation, distribution and reproduction in any medium or format, as long as you give appropriate credit to the original author(s) and the source, provide a link to the Creative Commons license, and indicate if changes were made. The images or other third party material in this article are included in the article's Creative Commons license, unless indicated otherwise in a credit line to the material. If material is not included in the article's Creative Commons license and your intended use is not permitted by statutory regulation or exceeds the permitted use, you will need to obtain permission directly from the copyright holder. To view a copy of this license, visit http://creativecommons. org/licenses/by/4.0/.

(c) The Author(s) 2021 